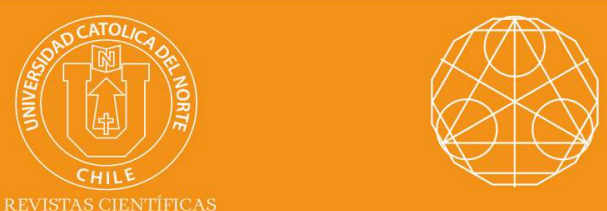

\title{
On star coloring of degree splitting of join graphs
}

S. Ulagammal*

Vernold Vivin J.* (iD orcid.org/0000-0002-3027-2010

*University College of Engineering, (Anna U. Constitutent College), Nagercoil, TN, India.

$\checkmark$ ulagammal2877@gmail.com

** University College of Engineering, (Anna U. Constitutent College), Nagercoil, TN, India.

$\checkmark$ vernoldvivin@yahoo.in

Received: October 2018 | Accepted: April 2019

\section{Abstract:}

A star coloring of a graph $G$ is a proper vertex coloring in which every path on four vertices in $G$ is not bicolored. The star chromatic number $\chi_{\mathcal{L}}(G)$ of $G$ is the least number of colors needed to star color $G$. In this paper, we have generalized the star chromatic number of degree splitting of join of any two graph $G$ and $H$ denoted by $G+H$, where $G$ is a path graph and $H$ is any simple graph. Also, we determine the star chromatic number for degree splitting of join of path graph $G$ of order $m$ with path $P_{n}$, complete graph $K_{n}$ and cyclevgraph $C_{n}$.

Keywords: Star coloring; Complete graph; Path and cycle.

MSC (2010): 05C15, 05C75.

Cite this article as (IEEE citation style):

S. Ulagammal and Vernold Vivin J., "On star coloring of degree splitting of join graphs", Proyecciones (Antofagasta, On line), vol. 38, no. 5, pp. 1071-1080, Dec. 2019, doi: 10.22199/issn.0717-62792019-05-0069. [Accessed dd-mm-yyyy]

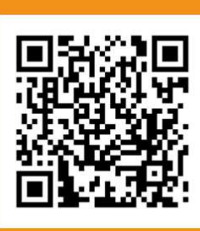

Article copyright: (C) 2019 S. Ulagammal and Vernold Vivin J. This is an open access article distributed under the terms of the Creative Commons Licence, which permits unrestricted use and distribution provided the original author and source are credited.

(cc) BY 


\section{Introduction}

All graphs in this paper are finite, simple, connected and undirected graph in $[2,3,7]$. The concept of star chromatic number was introduced by Branko Grunbaum in 1973. A star coloring $[1,5,6]$ of a graph $G$ is a proper vertex coloring in which every path on four vertices uses at least three distinct colors. Equivalently, in a star coloring, the induced subgraph formed by the vertices of any two colors has connected components that are star graph. The star chromatic number $\chi_{s}(G)$ of $G$ is the least number of colors needed to star color $G$.

Guillaume Fertin et al. [5] determined the star chromatic number of trees, cycles, complete bipartite graphs, outer planar graphs and 2dimensional grids. They also investigated and gave bounds for the star chromatic number of other families of graphs, such as planar graphs, hypercubes, graphs with bounded treewidth and cubic graphs and planar graphs with high - girth.

Albertson et al. [1] showed that it is NP-complete to determine whether $\chi_{s}(G) \leq 3$, even when $G$ is a graph that is both planar and bipartite. Coleman et al. [4] proved that star coloring remains NP-hard problem even on bipartite graphs.

For a given graph $G=(V(G), E(G))$ with $V(G)=S_{1} \cup S_{2} \cup S_{3} \cup$ $\ldots S_{t} \cup T$ where each $S_{i}$ is a set of all vertices of the same degree with at least two elements and $T=V(G)-\bigcup_{i=1}^{t} S_{i}$. The degree splitting graph $[8,9]$ of $G$, denoted by $D S(G)$, is obtained by adding vertices $w_{1}, w_{2}, \ldots w_{t}$ and joining $w_{i}$ to each vertex of $S_{i}$ for $1 \leq i \leq t$.

Let $G_{1}$ and $G_{2}$ be two graphs having disjoint point sets $V_{1}$ and $V_{2}$ and line sets $E_{1}$ and $E_{2}$ respectively. Then their join [7] is denoted by $G_{1}+G_{2}$ consists of $G_{1} \cup G_{2}$ and all lines joining $V_{1}$ with $V_{2}$.

\section{Main Results}

In the following section, we generalize the star chromatic number of degree splitting of join of any two graph $G$ and $H$ denoted by $G+H$, where $G$ is a path graph and $H$ is any simple graph. Also, we find the star chromatic number for degree splitting of join of path graph $G$ of order $m$ with path $P_{n}$, complete graph $K_{n}$ and cycle graph $C_{n}$. 


\subsection{Generalization of star coloring for degree splitting of join of path graph with any simple graph}

Theorem 2.1. Let $G$ be a path graph of order $m \geq 4$, and $H$ be any simple graph of order $n \geq 3$ then

$$
\chi_{s}(D S(G+H))=n+4 .
$$

Proof. Let $V(G)=\left\{u_{1}, u_{2}, u_{3}, \ldots, u_{m}\right\}$ and $V(H)=\left\{v_{1}, v_{2}, v_{3}, \ldots, v_{n}\right\}$. By definition of join graph, $G+H$ consists of $G \cup H$ and all lines joining $V(G)$ with $V(H)$. Thus we have $V(G+H)=\left\{u_{i}: 1 \leq i \leq m\right\} \cup$ $\left\{v_{j}: 1 \leq j \leq n\right\}=S_{1} \cup S_{2} \cup \ldots \cup S_{t}$ where $S_{1}=\left\{u_{i}: 1 \leq i \leq m\right\} \ldots S_{t}=$ $\left\{v_{j}: 1 \leq j \leq n\right\}$. To obtain $D S(G+H)$ from $G+H$, we add vertices $w_{1}, w_{2}, \ldots, w_{t}$ corresponding to $S_{1}, S_{2}, S_{3}, \ldots, S_{t}$ respectively. Thus $V(D S(G+$ $H))=V(G+H) \cup\left\{w_{1}, w_{2}, w_{3} \ldots, w_{t}\right\}$

We define a function $\sigma: V \rightarrow\{1,2, \ldots\}$. Now, we assign $n+4$ coloring $\sigma$ of degree splitting for join graph $(G+H)$ is defined as follows:

For every $1 \leq i \leq m$, we now distinguish $m$ as three cases:

Case(i): When $m \equiv 3(\bmod 3)$.

$$
\begin{gathered}
\sigma\left(u_{3 k-2}\right)=1, \text { for } 1 \leq k \leq \frac{m}{3} \\
\sigma\left(u_{3 k-1}\right)=2, \text { for } 1 \leq k \leq \frac{m}{3} \\
\sigma\left(u_{3 k}\right)=3, \text { for } 1 \leq k \leq \frac{m}{3}
\end{gathered}
$$

and for every $1 \leq j \leq n$, assign

$$
\sigma\left(v_{j}\right)=j+3
$$

and also assign

$$
\sigma\left(w_{1}\right)=\sigma\left(w_{2}\right)=\cdots=\sigma\left(w_{t}\right)=n+4 .
$$

Case(ii): When $m \equiv 1(\bmod 3)$.

$$
\sigma\left(u_{3 k-2}\right)=1, \text { for } 1 \leq k \leq\left\lceil\frac{m}{3}\right\rceil
$$




$$
\begin{gathered}
\sigma\left(u_{3 k-1}\right)=2, \text { for } 1 \leq k \leq\left\lfloor\frac{m}{3}\right\rfloor \\
\sigma\left(u_{3 k}\right)=3, \text { for } \quad 1 \leq k \leq\left\lfloor\frac{m}{3}\right\rfloor
\end{gathered}
$$

and for every $1 \leq j \leq n$, assign

$$
\sigma\left(v_{j}\right)=j+3
$$

and also assign

$$
\sigma\left(w_{1}\right)=\sigma\left(w_{2}\right)=\cdots=\sigma\left(w_{t}\right)=n+4 .
$$

Case(iii): When $m \equiv 2(\bmod 3)$.

$$
\begin{gathered}
\sigma\left(u_{3 k-2}\right)=1, \text { for } 1 \leq k \leq\left\lceil\frac{m}{3}\right\rceil \\
\sigma\left(u_{3 k-1}\right)=2, \text { for } \quad 1 \leq k \leq\left\lceil\frac{m}{3}\right\rceil \\
\sigma\left(u_{3 k}\right)=3, \text { for } \quad 1 \leq k \leq\left\lfloor\frac{m}{3}\right\rfloor
\end{gathered}
$$

and for every $1 \leq j \leq n$, assign

$$
\sigma\left(v_{j}\right)=j+3
$$

and also assign

$$
\sigma\left(w_{1}\right)=\sigma\left(w_{2}\right)=\cdots=\sigma\left(w_{t}\right)=n+4 .
$$

Therefore $\chi_{s}(D S(G+H)) \leq n+4$.

Now, we prove that $\chi_{s}(D S(G+H)) \geq n+4$. For this, we must prove that any coloring with $n+3$ colors will give us at least one bicolored cycle of length 4 . Let $S_{m}$ be the set colors used to color the vertices of $V(G)$ and let $S_{n}$ be the set of colors used to color the vertices of $V(H)$ then $S_{m} \cap S_{n}=\phi$. Since all possible edges exist between vertices of $V(G)$ and the vertices of $V(H)$. Suppose that we use $n+3$ colors for the vertices of $D S(G+H)$ then there exists at least one bicolored cycle of length 4 . Which is a contradiction for proper star coloring. Thus no coloring that uses $n+3$ colors can be a star coloring. Thus star coloring of $D S(G+H) \geq n+4$. Therefore $\chi_{s}(D S(G+H))=n+4$. This completes the proof. 
Corollary 2.2. Let $G$ be a path graph of order $m>4$ and $P_{n}$ be a path graph of order $n \geq 4$ and $m>n$ then

$$
\chi_{s}\left(D S\left(G+P_{n}\right)\right)=n+4 .
$$

Proof. We have $V\left(G+P_{n}\right)=\left\{u_{i}: 1 \leq i \leq m\right\} \cup\left\{v_{j}: 1 \leq j \leq n\right\}=$ $S_{1} \cup S_{2} \cup S_{3}$ where $S_{1}=\left\{u_{1}, u_{m}\right\}$ and $S_{2}=\left\{u_{i}: 2 \leq i \leq m-1 ; v_{1}, v_{n}\right\}$ $S_{3}=\left\{v_{j}: 2 \leq j \leq n-1\right\}$. To obtain $D S\left(G+P_{n}\right)$ from $\left(G+P_{n}\right)$, we add three vertices $w_{1}, w_{2}$ and $w_{3}$ corresponding to $S_{1}, S_{2}$ and $S_{3}$ respectively. Thus we get $V\left(D S\left(G+P_{n}\right)\right)=V\left(G+P_{n}\right) \cup\left\{w_{1}, w_{2}, w_{3}\right\}$.

Now, we assign $n+4$ coloring $\sigma$ of degree splitting for join graph $\left(G+P_{n}\right)$ is defined as follows:

For every $1 \leq i \leq m$, we now distinguish $m$ as three cases:

Case(i): When $m \equiv 3(\bmod 3)$.

$$
\begin{gathered}
\sigma\left(u_{3 k-2}\right)=1, \text { for } 1 \leq k \leq \frac{m}{3} \\
\sigma\left(u_{3 k-1}\right)=2, \text { for } 1 \leq k \leq \frac{m}{3} \\
\sigma\left(u_{3 k}\right)=3, \text { for } 1 \leq k \leq \frac{m}{3}
\end{gathered}
$$

and for every $1 \leq j \leq n$, assign

$$
\sigma\left(v_{j}\right)=j+3
$$

and also assign

$$
\sigma\left(w_{1}\right)=\sigma\left(w_{2}\right)=\sigma\left(w_{3}\right)=n+4 .
$$

Case(ii): When $m \equiv 1(\bmod 3)$.

$$
\begin{gathered}
\sigma\left(u_{3 k-2}\right)=1, \text { for } \quad 1 \leq k \leq\left\lceil\frac{m}{3}\right\rceil \\
\sigma\left(u_{3 k-1}\right)=2, \text { for } \quad 1 \leq k \leq\left\lfloor\frac{m}{3}\right\rfloor \\
\sigma\left(u_{3 k}\right)=3, \text { for } 1 \leq k \leq\left\lfloor\frac{m}{3}\right\rfloor
\end{gathered}
$$


and for every $1 \leq j \leq n$, assign

$$
\sigma\left(v_{j}\right)=j+3
$$

and also assign

$$
\sigma\left(w_{1}\right)=\sigma\left(w_{2}\right)=\sigma\left(w_{3}\right)=n+4
$$

Case(iii): When $m \equiv 2(\bmod 3)$.

$$
\begin{gathered}
\sigma\left(u_{3 k-2}\right)=1, \text { for } 1 \leq k \leq\left\lceil\frac{m}{3}\right\rceil \\
\sigma\left(u_{3 k-1}\right)=2, \text { for } \quad 1 \leq k \leq\left\lceil\frac{m}{3}\right\rceil \\
\sigma\left(u_{3 k}\right)=3, \text { for } 1 \leq k \leq\left\lfloor\frac{m}{3}\right\rfloor
\end{gathered}
$$

and for every $1 \leq j \leq n$, assign

$$
\sigma\left(v_{j}\right)=j+3
$$

and also assign

$$
\sigma\left(w_{1}\right)=\sigma\left(w_{2}\right)=\sigma\left(w_{3}\right)=n+4 .
$$

Therefore $\chi_{s}\left(D S\left(G+P_{n}\right)\right) \leq n+4$.

Now, we prove that $\chi_{s}\left(D S\left(G+P_{n}\right)\right) \geq n+4$. Suppose that we use $n+3$ colors for the vertices of $D S\left(G+P_{n}\right)$ then there exist a cycle of length 4 which is bicolored, a contradiction for proper star coloring. Thus no coloring that uses $n+3$ colors can be a star coloring. Thus star coloring of $D S\left(G+P_{n}\right) \geq n+4$. Therefore $\chi_{s}\left(D S\left(G+P_{n}\right)\right)=n+4$. This completes the proof.

Corollary 2.3. Let $G$ be a path graph of order $m \geq 4$ and $K_{n}$ be a complete graph of order $n \geq 3$ then,

$$
\chi_{s}\left(D S\left(G+K_{n}\right)\right)=n+4 .
$$


Proof. We have $V\left(G+K_{n}\right)=\left\{u_{i}: 1 \leq i \leq m\right\} \cup\left\{v_{j}: 1 \leq j \leq n\right\}=$ $S_{1} \cup S_{2} \cup S_{3}$ where $S_{1}=\left\{u_{1}, u_{m}\right\}$ and $S_{2}=\left\{u_{i}: 2 \leq i \leq m-1\right\}$ and $S_{3}=\left\{v_{j}: 1 \leq j \leq n\right\}$. To obtain $D S\left(G+K_{n}\right)$ from $\left(G+K_{n}\right)$, we add three vertices $w_{1}, w_{2}$ and $w_{3}$ corresponding to $S_{1}, S_{2}$ and $S_{3}$ respectively. Thus we get $V\left(D S\left(G+K_{n}\right)\right)=V\left(G+K_{n}\right) \cup\left\{w_{1}, w_{2}, w_{3}\right\}$.

Now, we assign $n+4$ coloring $\sigma$ of degree splitting for join graph $\left(G+K_{n}\right)$ is defined as follows:

For every $1 \leq i \leq m$, we now distinguish $m$ as three cases:

Case(i): When $m \equiv 3(\bmod 3)$.

$$
\begin{gathered}
\sigma\left(u_{3 k-2}\right)=1, \text { for } 1 \leq k \leq \frac{m}{3} \\
\sigma\left(u_{3 k-1}\right)=2, \text { for } \quad 1 \leq k \leq \frac{m}{3} \\
\sigma\left(u_{3 k}\right)=3, \text { for } 1 \leq k \leq \frac{m}{3}
\end{gathered}
$$

and for every $1 \leq j \leq n$, assign

$$
\sigma\left(v_{j}\right)=j+3
$$

and also assign

$$
\sigma\left(w_{1}\right)=\sigma\left(w_{2}\right)=\sigma\left(w_{3}\right)=n+4 .
$$

Case(ii): When $m \equiv 1(\bmod 3)$.

$$
\begin{gathered}
\sigma\left(u_{3 k-2}\right)=1, \text { for } 1 \leq k \leq\left\lceil\frac{m}{3}\right\rceil \\
\sigma\left(u_{3 k-1}\right)=2, \text { for } \quad 1 \leq k \leq\left\lfloor\frac{m}{3}\right\rfloor \\
\sigma\left(u_{3 k}\right)=3, \text { for } \quad 1 \leq k \leq\left\lfloor\frac{m}{3}\right\rfloor
\end{gathered}
$$

and for every $1 \leq j \leq n$, assign

$$
\sigma\left(v_{j}\right)=j+3
$$

and also assign

$$
\sigma\left(w_{1}\right)=\sigma\left(w_{2}\right)=\sigma\left(w_{3}\right)=n+4 .
$$


Case(iii): When $m \equiv 2(\bmod 3)$.

$$
\begin{gathered}
\sigma\left(u_{3 k-2}\right)=1, \text { for } 1 \leq k \leq\left\lceil\frac{m}{3}\right\rceil \\
\sigma\left(u_{3 k-1}\right)=2, \text { for } \quad 1 \leq k \leq\left\lceil\frac{m}{3}\right\rceil \\
\sigma\left(u_{3 k}\right)=3, \text { for } \quad 1 \leq k \leq\left\lfloor\frac{m}{3}\right\rfloor
\end{gathered}
$$

and for every $1 \leq j \leq n$, assign

$$
\sigma\left(v_{j}\right)=j+3
$$

and also assign

$$
\sigma\left(w_{1}\right)=\sigma\left(w_{2}\right)=\sigma\left(w_{3}\right)=n+4 .
$$

Therefore $\chi_{s}\left(D S\left(G+K_{n}\right)\right) \leq n+4$.

Now, we prove that $\chi_{s}\left(D S\left(G+K_{n}\right)\right) \geq n+4$. For this, we must prove that any coloring with $n+3$ colors will give us at least one bicolored cycle of length 4. Suppose that we use $n+3$ colors for the vertices of $\left(D S\left(G+K_{n}\right)\right)$ then there exists at least two adjacent vetices which receive the same color, a contradiction for proper coloring. Thus no coloring that uses $n+3$ colors can be a star coloring. Thus star coloring of $D S\left(G+K_{n}\right) \geq n+4$. Therefore $\chi_{s}\left(D S\left(G+K_{n}\right)\right)=n+4$. This completes the proof.

Corollary 2.4. Let $G$ be a path graph of order $m \geq 4$ and $C_{n}$ be a cycle graph of order $n \geq 3$ and $m>n$ then,

$$
\chi_{s}\left(D S\left(G+C_{n}\right)\right)=n+4 .
$$

Proof. We have $V\left(G+C_{n}\right)=\left\{u_{i}: 1 \leq i \leq m\right\} \cup\left\{v_{j}: 1 \leq j \leq n\right\}=$ $S_{1} \cup S_{2} \cup S_{3}$ where $S_{1}=\left\{u_{1}, u_{m}\right\}, S_{2}=\left\{v_{j}: 2 \leq j \leq m-1\right\}$, and $S_{3}=$ $\left\{v_{j}: 1 \leq j \leq n\right\}$. To obtain $D S\left(G+C_{n}\right)$ from $\left(G+C_{n}\right)$, we add three vertices $w_{1} w_{2}$, and $w_{3}$ corresponding to $S_{1}, S_{2}$ and $S_{3}$ respectively. Thus we get $V\left(D S\left(G+C_{n}\right)\right)=V\left(G+C_{n}\right) \cup\left\{w_{1}, w_{2}, w_{3}\right\}$.

Now, we assign $n+4$ coloring $\sigma$ of degree splitting for join graph $\left(G+C_{n}\right)$ is defined as follows:

For every $1 \leq i \leq m$, we now distinguish $m$ as three cases:

Case(i): When $m \equiv 3(\bmod 3)$. 


$$
\begin{gathered}
\sigma\left(u_{3 k-2}\right)=1, \text { for } \quad 1 \leq k \leq \frac{m}{3} \\
\sigma\left(u_{3 k-1}\right)=2, \text { for } \quad 1 \leq k \leq \frac{m}{3} \\
\sigma\left(u_{3 k}\right)=3, \text { for } \quad 1 \leq k \leq \frac{m}{3}
\end{gathered}
$$

and for every $1 \leq j \leq n$, assign

$$
\sigma\left(v_{j}\right)=j+3
$$

and also assign

$$
\sigma\left(w_{1}\right)=\sigma\left(w_{2}\right)=\sigma\left(w_{3}\right)=n+4 .
$$

Case(ii): When $m \equiv 1(\bmod 3)$.

$$
\begin{gathered}
\sigma\left(u_{3 k-2}\right)=1, \text { for } 1 \leq k \leq\left\lceil\frac{m}{3}\right\rceil \\
\sigma\left(u_{3 k-1}\right)=2, \text { for } \quad 1 \leq k \leq\left\lfloor\frac{m}{3}\right\rfloor \\
\sigma\left(u_{3 k}\right)=3, \text { for } \quad 1 \leq k \leq\left\lfloor\frac{m}{3}\right\rfloor
\end{gathered}
$$

and for every $1 \leq j \leq n$, assign

$$
\sigma\left(v_{j}\right)=j+3
$$

and also assign

$$
\sigma\left(w_{1}\right)=\sigma\left(w_{2}\right)=\sigma\left(w_{3}\right)=n+4 .
$$

Case(iii): When $m \equiv 2(\bmod 3)$.

$$
\begin{gathered}
\sigma\left(u_{3 k-2}\right)=1, \text { for } \quad 1 \leq k \leq\left\lceil\frac{m}{3}\right\rceil \\
\sigma\left(u_{3 k-1}\right)=2, \text { for } \quad 1 \leq k \leq\left\lceil\frac{m}{3}\right\rceil \\
\sigma\left(u_{3 k}\right)=3, \text { for } \quad 1 \leq k \leq\left\lfloor\frac{m}{3}\right\rfloor
\end{gathered}
$$


and for every $1 \leq j \leq n$, assign

$$
\sigma\left(v_{j}\right)=j+3
$$

and also assign

$$
\sigma\left(w_{1}\right)=\sigma\left(w_{2}\right)=\sigma\left(w_{3}\right)=n+4 .
$$

Therefore $\chi_{s}\left(D S\left(G+C_{n}\right)\right)=n+4$.

\section{Conclusion}

In this paper, we have generalized the star chromatic number of degree splitting of join of two graph $G$ and $H$ denoted by $G+H$, is $n+4$ where $G$ is a path graph and $H$ is any simple graph. Also we gave the exact values of the star chromatic number for degree splitting of join of path graph $G$ of order $m$ with path $P_{n}$, complete graph $K_{n}$ and cycle $C_{n}$ repectively.

\section{References}

[1] M. Albertson, G. Chappell, H. Kierstead, A. Kündgen and R. Ramamurthi, "Coloring with no 2-colored P4's", The electronic journal of combinatorics, vol. 11, no. 1, 2004. [On line]. Available: https://bit.ly/2ttZpiC

[2] J. Bondy and U. Murty, Graph theory with applications. London: MacMillan, 1976.

[3] J. Clark and D. Holton, $A$ first look at graph theory. World Scientific, 1969.

[4] T. Coleman, J. Moré, "Estimation of sparse Hessian matrices and graph coloring problems", Mathematical programming, vol. 28, no. 3, pp. 243-270, Oct. 1984, doi: 10.1007/BF02612334.

[5] G. Fertin, A. Raspaud and B. Reed, "Star coloring of graphs", Journal of graph theory, vol. 47, no. 3, pp. 163-182, Aug. 2004, doi: 10.1002/jgt.20029.

[6] B. Grünbaum, "Acyclic colorings of planar graphs", Israel journal of mathematics, vol. 14, no. 4, pp. 390-408, Dec. 1973, doi: 10.1007/BF02764716.

[7] F. Harary, Graph theory. New Delhi, Narosa, 1969.

[8] R. Ponraj and S. Somasundaram, "On the degree splitting graph of a graph", National academy science letters, vol. 27, no. 7-8, pp. 275-278, 2004.

[9] E. Sampathkumar and H. Walikar, "On splitting graph of a graph", Journal of Karnatak University Science, vol. 25-26, pp. 13-16, 1981. [On line]. Available: https://bit.ly/2Er10HZ 